\title{
Elementary student achievement and teacher perception of an advanced chemistry curriculum
}

\author{
Daniel B. Fried ${ }^{1}$, Pablo P.L. Tinio ${ }^{2}$,Azuri Hughes ${ }^{1}$, Diana Paneque ${ }^{1}$ \\ ${ }^{1}$ Department of Chemistry, Saint Peter's University, Jersey City, New Jersey, USA \\ ${ }^{2}$ Department of Educational Foundations, Montclair State University, Montclair, New Jersey, USA
}

\begin{abstract}
:
Studying nature from the atomic and molecular perspective gives students access to modern scientific paradigms and could improve achievement and motivation towards science learning. A visual and tactile approach to chemistry can form the foundation for a child-friendly but high-level chemistry curriculum and could be a powerful tool to invigorate early STEM learning. This five-session study utilized a previously reported organic chemistry learning program that featured animated presentations, hands-on molecular model building, and image-based computer modeling of macromolecules. Twenty-six fourth, fifth, and sixth grade students participated in the study. Importantly, teachers at the school showed a high degree of engagement and participationtowards the program, and they embedded the chemistry material in their classes and projects. This teacher involvement created synergy with the program and supports the notion that high-level chemistry content can be very beneficial to students and teachers, and can open new avenues of study, even for young students. Teacher survey results revealed that the exposure to the chemistry learning program benefitted them professionally by providing them with useful knowledge and pedagogical strategies that they could readily use to enhance their own teaching.
\end{abstract}

Keywords: Organic chemistry, Molecular modeling, Teacher motivation

\section{Introduction}

As students progress through the elementary and middle school years, motivation towards STEM learning declines (Long, Monoi, Harper, Knoblauch, \& Murphy, 2007). This decline in motivation could lead to negative consequences for students later in life. For example, to be competitive in an increasingly complex and high-tech job market, individuals need to possess some level of scientific literacy. It also helps to have a positive attitude towards STEM so that one could consider a career in a STEM field. The erosion of interest and motivation towards STEM in the later years of school is therefore detrimental not only to the future lives of students, but also to the STEM fields in general. (Metz, 2008; National Academy of Sciences, 2007; Nord, et al., 2011). Importantly, research has shown that when students feel in control of their learning, and that the topics they learn are aligned with their own learning goals, the decline in their interest in STEM could be mitigated (Vedder-Weiss \&Fortus, 2012).

Our previous research has indicated that organic chemistry and biochemistry, despite their absence from traditional elementary school curricula, are well aligned with what students naturally want to study. These advanced subjects provide an empowering and engaging classroom experience for elementary students (Fried et. al., 2019). Building a strong foundation in thinking about the world at the atomic and molecular levels could influence what careers students choose to pursue. Furthermore, studying biochemistry prepares students for success in STEM fields and could help them make more educated decisions regarding environmental, nutritional, and health issues in their own lives and communities. 
Although topics from organic and biochemistry resonate well with young students who naturally want to understand more about the world, promoting meaningful understanding of such high-level topics requires the use of specific pedagogical supports, such as modeling tools, that help students visualize and understand otherwise invisible phenomena. Modeling activities are therefore essential to a chemistry learning program (Dori \& Kaberman, 2012). Experiencing modeling activities that take advantage of current technologies, such as computerized molecular modeling, not only benefit students, but also serve as examples for instructors to improve daily teaching activities (Kali \& Linn, 2008). Molecular modeling software allows detailed, experimentally-derived models to be implemented, and allows the teacher to hide or show structural information depending on what he or she is trying to present. By focusing the learner's attention in this way, critical information can be made salient, saving the learner time and energy as they interpret new content. Teaching tools, not traditionally used with children, such as the professional-grade modeling software PyMol (PyMol, Schrödinger LLC), were therefore used in our study to render biomolecules in a variety of ways.This allowed for the creation of images that were appropriate for teaching even young children.

There are several general principles that can be used to design curricula that develop visuospatial chemistry thinking ( $\mathrm{Wu} \&$ Shah, 2004). One such principle involves emphasizing strong links between the three-dimensional nature of molecules and their two-dimensional representations. Another principle involves the use of teaching materials to "reduce cognitive load by making information explicit." The methodology employed by our previous and current research strived to, as explicitly as possible, trace every mental step required to interpret chemical nomenclature, connect element atomic structure to properties, and understand and apply chemical concepts such as charge, bonding, and geometry. This was done both by rendering appropriate PyMol images of biomolecules as well as by being consistent and thoughtful with how all chemical information was presented.

In our previous work (Fried et. al., 2019), we showed that a highly visual and tactile classroom learning environment allowed students in grades 3-5 to engage in organic chemistry learning to a surprising level after just 3.5 hours of a teaching intervention. In keeping with other studies, we confirmed that a curriculum built on rich, meaningful, and interconnected scientific concepts can provide students with a forum to pose sophisticated questions and can pique their interest in learning (Clements \&Sarama, 2016).

It is important to note that the present and previously reported study differ in several respects. The previous study was conducted in an urban, public school while the current study was conducted in a private Montessori school, which allowed more time for the study because of the more flexible curriculum. The additional time allowed for a more relaxed pace of instruction as well as more actual instruction hours (7.5 vs. 3.5 hours). The program took place over five weekly, 90-minute classroom sessions. A significant difference between this and our previous study was the high level of involvement of the teachers. With the greater curricular flexibility inherent in a private school environment, teachers took time to review and apply the content of the study between the weekly sessions. While the content covered in the current study was identical to the previous study, the curriculum was completely embedded in the academic life of the students for many weeks. During the time between the PI-led sessions, students practiced building chemical models with model kits, reviewed concepts using learning puzzles, and with the help of the teachers, invoked content from the program during their own classroom activities and field trips. In particular, a water quality field trip that was led by the teachers, and that investigated the Hudson River, gave students extensive practice with applying concepts from the chemistry sessions. The close involvement of the teachers showed that the chemistry enrichment program benefits not only the students, but also gives teachers useful pedagogical understanding and tools that can motivate them to enhance their own teaching efforts, and even enjoy teaching more (Daniels, 2017; Martin, 2006). 
A main expectation for this study was that students would achieve greater content learning and motivation due to the more extended program and the greater involvement of the teachers. It was also expected that the regular teacher-led classes would be positively influenced by the ongoing chemistry enrichment program, and that teachers might be able to delve deeper into some concepts that were supported by the program materials. The study included the following data sources: a preclass student survey, in-class observations, a final assessment for content learning, a qualitative final assessment of student perceptions regarding the program, and a teacher survey of perceptions about the program and their own teaching practice. This last element involving teacher perceptions furtherextends the previous study, which focused only on students.

\section{Methods}

\section{Participants}

Twenty-six students, comprising a mixed-age classroom at a Montessori school, were selected for the study. Eleven participants were 4th graders, 9 participants were 5th graders, and 6 participants were 6 th graders. The class comprised the entire upper elementary class, and no students were excluded for participation in the study. Five teachers from the school, all of whom taught the student participants or supervised their curriculum, were also included in the study.All elements of the study were approved by the Institutional Review Board of Saint Peter's University.

\section{Program Curriculum and Procedures}

All instruction, led by the PI with the classroom teachers present,consisted of five 90-minute sessions for a total of about 7.5 hours of contact time. The program included the same content as in the previous study, but with about twice as much classroom time, which allowed more time for review, classroom discussions, and practice.

Details about the program's content and methods appear below.

A pre-class survey was first given to the students. The surveys asked about fundamental chemistry concepts such as the meanings of the words atom and molecule. Periodic tables were handed out to the students and they used colored pencils to shade in the elements most commonly found in biochemistry: hydrogen, carbon, nitrogen, and oxygen. The goal of the first class was to help students practice using the atom color scheme, and for them to discover patterns in atomic bonding. First, PyMol was used to view an x-ray structure of rhodopsin, the photo-active protein of the eye. Students engaged in a conversation about patterns that they observed in the protein structure. As they explored the 3D structure, they were asked to notice that atom color (element identity) was correlated with the number of bonds formed by that element. They began to record their observations, determining the rules of bonding themselves. They noticed other patterns as well, such as the presence of carbon rings, and that there were patterns in the order that atoms appeared in the protein backbone. As the lesson concluded, they were told that the goal of the course was to help them learn college-level concepts such as why the atoms bond together the way they do in the structure.

Later lessons used visualizations to help students connect an element's position on the periodic table with bonding behavior. Students engaged in learning units about organic chemistry nomenclature, fuel molecules, amino acids, and aroma molecules. Lessons connected with everyday experiences, and hand-held molecular modeling activities accompanied most lessons, providing an active learning environment for the entire class. Three-dimensional molecular modeling activities were also used to clarify concepts and to demonstrate how to properly use the hand-held molecular models. For example, PyMol was used to demonstrate the concept of chirality for the entire class so that each student could properly build and examine their chiral amino acid molecules. Many other in-hand chemical reactions were also performed. For example, a group activity involving the polymerization of amino acids allowed demonstration of the dehydration reaction concept, and working as a class, 
students built a long amino acid polymer. Experiencing this peptide polymerization reaction, students had the opportunity to more clearly understand protein primary structure. With a better understanding of how amino acids connect to form peptides, protein crystal structures were then reexamined in PyMol to show students how they have greatly improved their ability to understand and interpret such complex structures, compared with their ability at the beginning of the program.

\section{Detailed description of lessons}

The lessons featured model building using Molymod kits and activities based on molecular modeling with PyMol, as described previously. Child-friendly animated presentations guided classroom discussions and activities.

Day 1: In addition to the introductory modeling activity described above, the first session focused on gas molecules. Students familiarized themselves with single and double bonds by building models of hydrogen, oxygen, and nitrogen gas. PyMol-based visualization also introduced students to how oxygen binds to the iron in hemoglobin.

Day 2: Students learned about and built hydrogen-rich molecules such as methane, ammonia, and water, and built models of hydrocarbon fuels. They also performed a hand-held chemical reaction demonstrating the combustion of methane. The emphasis of the lesson was familiarization withcommon molecular geometries and typical bonding patterns of the elements.

Day 3: Students were introduced to the concept of the atomic nucleus and the class discussed visualizations showing the size and mass differences between atomic nuclei. These differences were experienced in a tactile lesson using density cubes, where students could feel the differences between samples of aluminum and tungsten. An animated periodic table visualization helped students draw simple atomic models that showed proton numbers and electron orbital configurations for the first 10 elements. These Lewis dot-like structures were also colored to help students connect element identity to electron orbital arrangement.

Day 4: Animated visualizations showed students how the structure of the outer electron orbital reveals the number of bonds that the element is capable of forming. Using the same Lewis dot-like diagrams, students practiced adding hydrogen atoms to carbon, nitrogen, oxygen, and fluorine. Students were challenged to apply the covalent bonding concept to other less familiar elements such as sulfur.

Day 5. Model building was used to reinforce the connections between an element's position in the periodic table and its bonding behavior. Two model building exercises were performed. First, students selected an aroma molecule from a panel of fruit and spice flavors. For example, students selected the drawing of isoamly acetate, which is the essence of banana, and built the structure using the molecular model kits. Students were encouraged to find patterns between flavor type and chemical structure. For example, spice flavors typically have a different structure than fruit flavors. The second building activity prompted each student to build an ethylene molecule. Students then viewed a visualization that showed how the molecule could undergo a radical polymerization to form polyethylene plastic. Students modeled this reaction by breaking one of the ethylene's bonds and forming new bonds with their classmates' molecules. The result of this group activity was the creation of a several-feet-long plastic polymer.

Day 6. Students were introduced to the acid/base concept and its connection to molecular charge. Animated presentations showed students how certain lone pair electrons of a basic molecule could attract a proton, and how some protons could be removed from an acid. Students built structures of the 20 amino acids and practiced removing and adding acidic protons. Students also observed the 
differences in amino acid side chain size and acid/base activity. Students practiced identifying amino acid side chains on a protein structure rendered in PyMol. The final assessment was administered at the end of the session.

Classroom discussions were based on animated presentations that provided a visual framework for chemistry. In particular, to help students predict bonding behavior of elements, an interactive periodic table in which element boxes could be colorized was used in conjunction with Lewis-dot structure-like atom/molecule diagrams. Figure 1 shows an example of how the color-coded visualizations connect the periodic table, bonding, and chemical nomenclatures. For carbon (element symbol C), element symbol boxes, protons, and electrons have been shaded dark gray to show how proton number (six for carbon) matches element number and how electron shell occupancy corresponds with the number of elements on each row. For carbon, two elements on the first row and four elements on the second row are shaded dark gray; this corresponds with two electrons in the inner shell and four electrons in the outer shell. The visualization allows students to see why carbon, with its four unpaired outer electrons, binds with four hydrogen atoms (light gray). The resulting methane molecule can be represented by the Lewis-dot structure-like model to the left, a ball-andstick model rendered in PyMol to the right, or a chemical drawing showing atom symbols in the center.

The nomenclatures described here form the foundation for student understanding of chemical bonding, and iterative examples throughout the course build familiarity and confidence in the use of the periodic table. Throughout the program, students became very familiar with the diagrams and nomenclatures below, as well as with the process by which element bonding could be predicted using the periodic table. Students also physically built molecules using molecular model kits, giving them an active learning opportunity to practice constructing and visualizing molecular structures.

The content and instructional methods used in this study were the same as in the previous study (Fried et al., 2019), but about twice as much classroom instruction time was allocated for the programming. Additionally, chemistry learning resources were made accessible to students so that they could practice chemistry concepts during theirfree time. The classroom was provided with the following manipulatives: four instructor-sized Molymod molecular model kits; PyMol computer molecular modeling software installed on classroom desktops; and chemistry review puzzles, created specifically for the class. The review puzzles consisted of cards that reviewed content and chemical structures introduced during the sessions. 


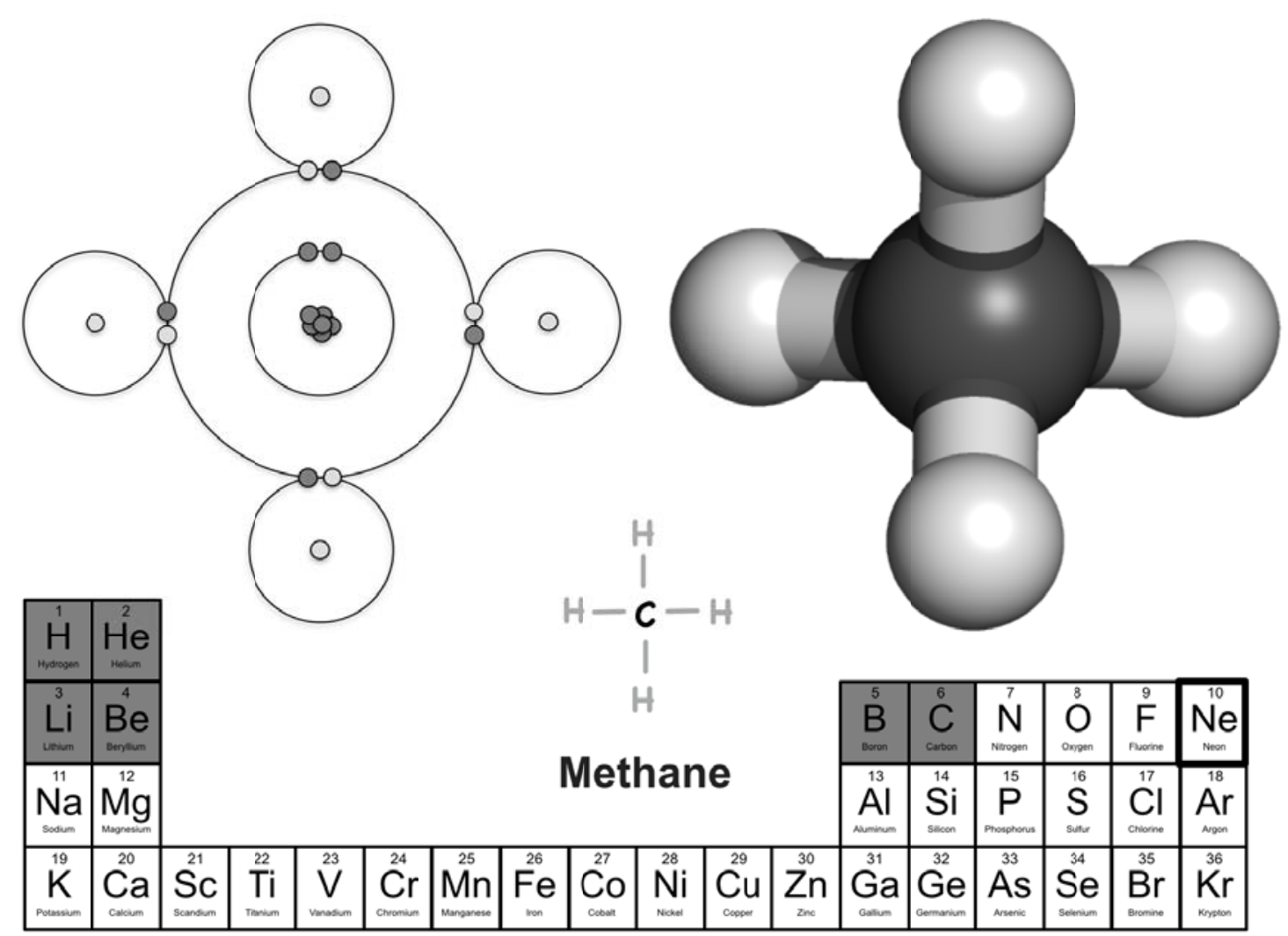

Figure 1. Example slide from the day 1 lesson.An annotated periodic table guides students in connecting element number and location to bonding. Here students can see methane represented first by a Lewis dot structure-like representation (left) a three-dimensional covalently bound molecule, rendered in PyMol (right) and a chemical drawing showing element symbols and bonds (center).

The program was bolstered by close involvement of the teachers. Classroom teachers and other faculty from the school became very interested in the program, and on their own, began to incorporate concepts from the sessions into their regular teaching activities. This provided students with even more practice and application of the material. This synergy between the chemistry program and classroom teachers was somewhat unexpected, but increased student involvement and enthusiasm for chemistry learning.

\section{Results}

Pre-class Student Survey

A survey consisting of the following questions was given to the class before the program began. The questions were designed to assess students' perceptions about STEM learning in general and chemistry in particular. It is important to note that we did not administer a formal pre-test that specifically assessed the students' knowledge of the chemistry concepts to be taught, because based on the previous study (Fried et al., 2019), and the PI's experiences with implementing the program in others schools, knowledge of general chemistry, organic chemistry, and biochemistry in primary grade levels is at floor level. The typical elementary, and often high school, curricula do not deal with these topics. Student responses to the pre-class student survey are summarized below.

1. What would you like to learn about in science classes in the future?

$20 \%$ of students said they wanted to learn more about elements.

$16 \%$ of students said they wanted to learn more about the periodic table.

$28 \%$ of students wanted to know about how chemical reactions or explosions work.

Some students had high level questions about science: "I would like to learn about how atoms 
first formed on Earth." "I would like to learn about the different ways to make certain chemicals and molecules." "How does chemistry work?" "[I want to learn] functions of the brain, enzymes, and proteins.

Comparing what students wanted to learn with what students already knew revealed a large knowledge gap. The following questions show that background knowledge about chemistry was, as expected, minimal.

2. What is an atom? Write a sentence stating what you know about atoms.

$24 \%$ simply stated that atoms were small.

$20 \%$ said that they were building blocks of matter.

$12 \%$ said they make up everything in the body.

$4 \%$ said they were particles.

$20 \%$ said they "needed help."

Only $16 \%$ of students gave responses that showed familiarity with the essential nature of atoms, that they make up molecules, or that they contain protons, neutrons, and electrons.

3. Give an example of an element.

While $52 \%$ of students could name at least one element from the periodic table, such as helium, iron, or tungsten, $22 \%$ of students named classical elements, such as fire, water, and earth.

$26 \%$ left the question blank.

4. What is a molecule? Write a sentence stating what you know about molecules.

Only $20 \%$ of student responses indicated understanding that molecules were made of atoms. The remaining responses showed great confusion about what molecules were. "A molecule makes up an atom." "A molecule is a water cell." "A molecule is made up of cells." "Molecules are cells that build up a person."

5. Give an example of a molecule.

$56 \%$ of students left this question blank. The only molecule that was named was water, named by $24 \%$ of students.

$\square$ Other students were confused about the meaning of the word molecule, with $8 \%$ of students giving examples of atoms, rather than molecules, and $12 \%$ of students stating that cells or amoebas were examples of molecules.

6. What do you want to be when you grow up?

$24 \%$ of students named STEM-area careers, while $36 \%$ of students were undecided.

7. On a scale of 0 to 10 , how interested in science classes are you?

The overall class average was 7.5, indicating a high interest in science learning.

In-class Observations

Students were extremely enthusiastic throughout the program and showed high levels of participation during classroom discussions and activities. They looked forward to the sessions and practiced the material between the weekly sessions. Students practiced drawing atomic and molecular diagrams, as well as building organic molecules using the model kits housed in the school's STEM classroom. One unit of the program that was particularly popular with the students was flavor molecules. In their spare time, students used the model kits and worksheets to build fruit aroma esters such as those associated with banana, pear, apple, and pineapple. Two classes featured polymer building activities that offered opportunities for students to have fun working together to construct models of polyethylene plastic and polyhydroxybutyrate, a biodegradable plastic. 
Throughout the program, classes were highly interactive with student questions often guiding instruction and activities.

\section{Final Assessment of Content Learning}

Twelve final assessment questions gauged student learning from the program. The final assessment was identical to that of our previous study. Descriptions of the questions are summarized below.

Questions 1-3 assessed students' ability to work with a Lewis structure-like atomic diagram. For question 1, students were asked to determine the identity of an oxygen atom diagram by examining electron configuration. For question 2, students were asked to create a covalently-bonded molecule from the atom diagram, by adding hydrogen atoms and creating share pairs of electrons. For question 3 , students were asked to name the resulting water molecule.

Questions 4-5 probed student understanding of organic chemistry nomenclature. They were presented with a carbon skeleton diagram of vanillin, the main aroma molecule for vanilla bean. Question 4 checked student understanding of the carbon skeleton nomenclature, by asking students to add bonded hydrogen atoms to the structure. Question 5 asked students to further interpret their finalized structure, count all the atoms, and write a molecular formula, $\mathrm{C}_{8} \mathrm{H}_{8} \mathrm{O}_{3}$.

Questions 6 and 7 presented students with the structure of acetic acid, although one oxygen atom incorrectly contained three bonds, an extra hydrogen. Students were asked to identify and correct the mistake.

Question 8 asked students to draw a structure for the formula $\mathrm{C}_{2} \mathrm{H}_{7} \mathrm{~N}$. Two correct structures were possible, dimethylamine or ethylamine. Question 9 asked students do draw any legitimate covalent structure, without giving a formula prompt.

Questions 10-12 enabled students to demonstrate their ability to infer the binding properties of elements that were not discussed previously in the program. For question 10, students were asked to interpret the periodic table and draw an atomic diagram for phosphorus, showing the proper number of orbitals and paired and unpaired electrons. For question 11, students were asked to state how many bonds they thought phosphorus was capable of forming. And finally for question 12, in a similar manner to question 2, students created a covalently-bonded structure by adding atoms of their choice to create a hypothetical phosphorus-containing molecule.

As indicated in Figure 2, students were highly successful at answering the questions on the final assessment.

All 12 questions were answered correctly by at least $75 \%$ of 6 th graders.

Several questions were answered correctly by $100 \%$ of the students. For example, all 4 th and 6 th graders were able to complete a covalent bonding diagram of a water molecule, and all 5 th and 6 th graders were able to correct a mistake in the structure of acetic acid. The majority of students were even able to properly answer the challenging questions about the atomic structure and bonding properties of phosphorus.

$\square \quad$ The lowest scoring question was question 12, which asked students to demonstrate the bonding of phosphorus, but still, the majority of students succeeded in answering the question correctly.

Qualitative Student PerceptionsAssessment

A final assessment consisting of the following questions was given to the class at the conclusion of the program. Student responses are summarized below. 
1. In the class, what did you like learning about the most?

Students were enthusiastic about the core features of the class. Many students reported that learning about bonds was their favorite topic. Others mentioned the periodic table, molecules, formulas, "acids and how they are made," and protons. One student said they liked "everything."

2. What did it feel like to learn college science? Were you surprised you could do it? Did it feel easy or hard?

The fact that students were aware that they were learning college science gave them a special connection to the class. One student said it was "good but hard" to learn college science. Several students mentioned they were surprised they could do it: "It was easy, [and I was] surprised I could do it." Another said "It didn't feel easy or hard, it felt medium." Only one student wrote that the material was "too hard." Other students said it was "amazing" and that they were "honored."

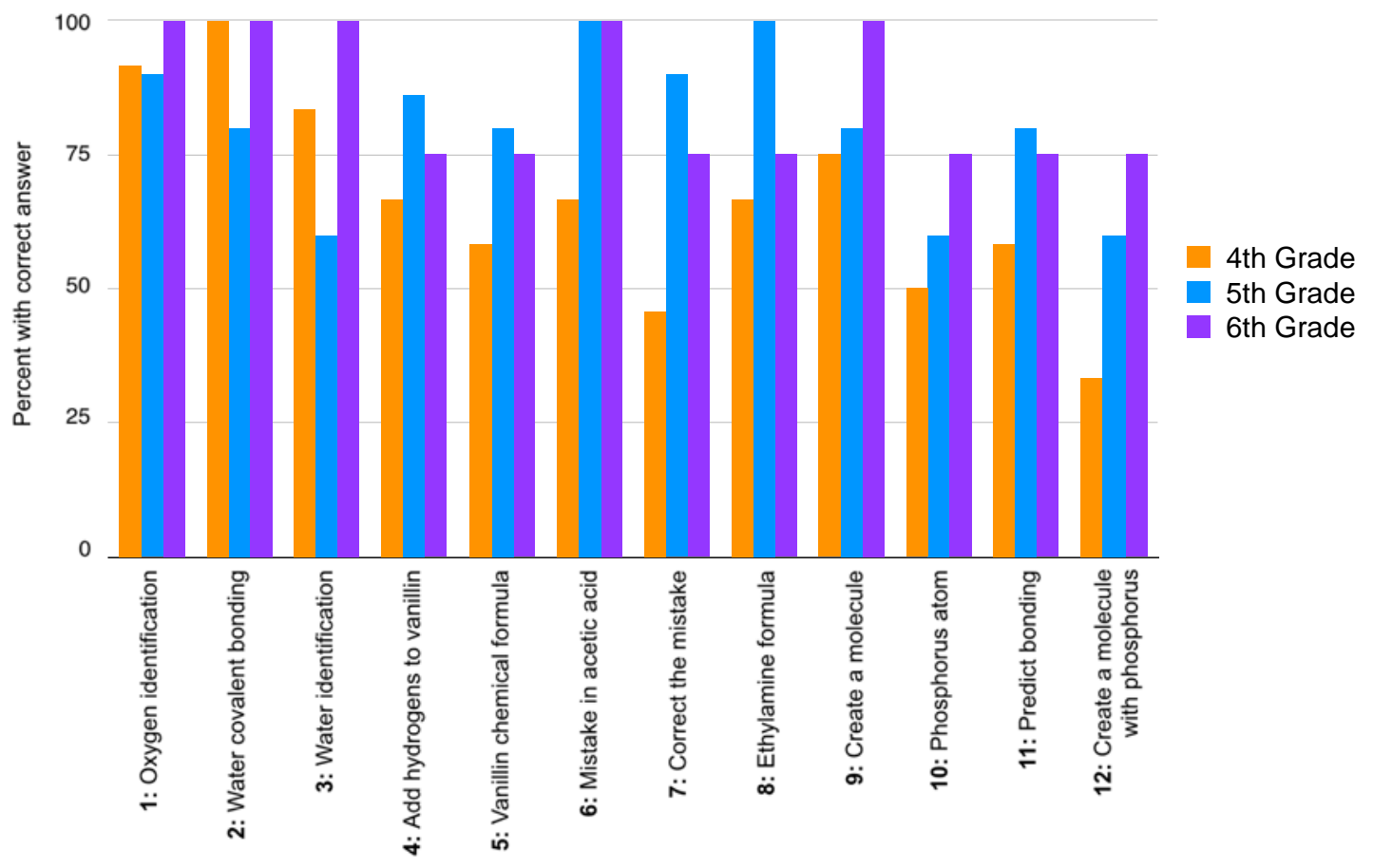

Figure 2. Percent of students with correct answers for each of the twelve assessment questions.

3. What other science questions do you have? What else would you like to learn in future classes?

Below are some answers about what students wanted to learn in the future.

"I would like to learn how to memorize the acids. Why do people have acids in them? Won't the acids burn them?"

"I want to learn more about what we are learning already."

"I would like to learn everything about chemistry."

"I would like to learn more about what's in things. For example, what atoms are in glass?"

Teacher Perceptions Survey

In the survey, teachers were asked for their opinions about high-level science being taught at the elementary level before and after experiencing the program. They were asked to report on feedback about the program from students and parents as well as any socioemotional and academic changes 
that they observed in the students after participation in the program. Teachers were also asked about their overall evaluation of the program and whether the program influenced their teaching of STEM subjects.

Overall, teachers were very impressed by student learning and enthusiasm during the program. Most felt that the program validated many of the ideas about teaching that they had-that students can be challenged with material typically considered above grade level and that hands-on and active learning sessions can help kids with different approaches to learning. Several teachers also mentioned the extra motivation that students brought to the program, especially when they became aware that they were studying college-level material. Teachers also reported that parents were excited about their children's participation in the program. Some teacher responses mentioned how they were able to draw on the unique content from the program to enhance their own instruction.

"As a STEM educator, it is important for me to always braid in and help make those connections for students. [The program] enabled and helped enhance this meta-discipline. I can draw on other concepts and experiences and root them back to the foundation. An example would be to explain why water and oil do not mix. With their background knowledge of molecular composition, they are able to connect and understand why it behaves as it does."

"We all learned from it, students and teachers alike. The students were so excited and absorbed the content like sponges. The way the class was taught and the content of the curriculum was spot-on for the students to gain understanding with the materials."

In answering the question about what could be improved about the program, several teachers suggested slowing down the classes to give more time for the hands-on activities and practice. Teachers agreed that more frequent and more extensive programming would be beneficial to the students.

\section{Discussion}

This chemistry learning program was designed to help elementary students interrogate some of the big questions of modern science. What are atoms and molecules? What are their structures? How do elements from the periodic table combine to form new materials? Pre-class survey questions showed that students really do think about these questions, and they hope that their school science classes can help them find answers. The program curriculumused atomic and molecular modeling tools to give students a foundation in the periodic table, atomic structure, and chemical bonding. Access to these topics provided students with a foundation on which to begin their study of the physical and life sciences, and helped to motivate their learning. The curriculum bridged the gap between what students wanted to learn and what they could learn. In addition to creating a fun, unique, and rewarding learning environment, the program also resulted in strong content learning. The class as a whole scored highly on questions dealing with chemical nomenclature, atomic structure, covalent bonding, and interpretation of the periodic table-topics that would also appear on high school and college chemistry exams. Students and their teachers indicated that because the learning was "college level," students seemed even more highly motivated to achieve; they gained gratification and pride from being able to learn. The high-level questions posed by students during the classes and in the final student perceptions assessment showed that students were willing and able to learn highly advanced material.

While high-level chemistry is not typically part of an elementary school education, the material resonated well with students, and the visual and tactile methods created a kid-friendly setting in which to learn. Student achievement, as measured through program-specific content assessment, 
showed that students became competent in many of the most important areas of chemistry, such as being able to connect the periodic table with electron orbital structure, as well as the ability to interpret and improvise two-dimensional and three-dimensional chemical diagrams. Such knowledge forms the basis for a chemistry literacy that can have far-reaching academic benefits for the students. Additionally, as in our previous study (Fried et al., 2019), the program excited students about STEM learning and motivated them to seek out more knowledge.

Another important aspect of the study is that it took place at a Montessori school where students have been, from an early age, encouraged to take their education into their own hands, ask questions, have discussions, and work collaboratively. The idea of using manipulatives to learn was familiar to the students, and they felt free to use them. Students were used to working at learning stations in their free time, so the model sets and learning puzzles made available between program sessions, were utilized often by the students. The freedom that teachers had in adjusting the curriculum to meet the interests of the students also had a positive effect on the study; the chemistry curriculum introduced in the study was frequently drawn on throughout the week by teachers, and students were likely better able to retain the information with this reinforcement.

Survey results from five teachers who had contact with the program and its participants reveal strong commitment to the methodology and indicated that exposure to the chemistry content will have a lasting effect on their educational outlook and teaching. In future implementations of this curriculum, teachers will play a larger part, and the curriculum needs to be piloted with teacher-led, rather than PI-led, classes. This program showed that teachers believe in the effectiveness and positive benefits of the program, and they report strong support for embedding the advanced chemistry curriculum into their classes. Teachers felt that the program greatly benefited their students, but they also reported that it helped clarify certain topics for their own teaching, and that there was synergy between the chemistry curriculum and other learning units in the students' normal curriculum.

In addition, the teachers who were observed or participated in the classes reported a boost in their own confidence in teaching science. The class helped them review foundational chemistry concepts and also gave them enrichment and introduced new content that gave a fresh perspective on the subject. Since their students had been exposed to these chemistry concepts as well, teachers were able to begin lessons at a higher level and could connect biology and environmental science to chemistry. This embedding of the chemistry in the daily STEM coursework of the class contributed to the high assessment scores of the students, as they continuously applied and practiced the material. One way to consider the teachers' experiences of the program is that it served as a type of extended professional development that provided teachers with more knowledge and pedagogical tools that helped them improve their practice and that boosted their motivation (Daniels, 2017; Martin, 2006). We hope that in the future, teachers can use elements from our curriculum to help bolster learning in many areas of STEM.

Perhaps most importantly, this study showed that it is possible to provide students with high-level science content that answers the questions that they have about how the world works. Pre-class survey questions indicated a strong curiosity about the atomic and molecular world, what things are made of, and how chemical reactions work. Without a program in place to answer these questions, many of these students might have eventually lost motivation to learn science and could become frustrated that questions that are important to them were not being answered in school. Overall, this study demonstrated that a modeling and visualization-based chemistry curriculum can help bring a more sophisticated and rewarding level of STEM learning to elementary students. 


\section{Acknowledgements}

We would like to thank the Hudson Montessori School and its faculty for welcoming us into their schools and for their collaboration on the project.

\section{References}

Clements, D. H., \& Sarama, J. (2016). Math, science, and technology in the early grades. The Future of Children, 75-94.

Daniels, E. (2017). Curricular factors in middle school teachers' motivation to become and remain effective. RMLE Online, 40(5), 1-14.

Dori, Y. J., \& Kaberman, Z. (2012). Assessing high school chemistry students' modeling sub-skills in a computerized molecular modeling learning environment. Instructional Science, 40(1), 69-91. doi:10.1007/s11251-011-9172-7

Fried, D. B., Tinio, P. P. L., ...

Kali, Y., \& Linn, M. C. (2008). Designing effective visualizations for elementary school science. The elementary school journal, 109(2), 181-198.

Long, J. F., Monoi, S., Harper, B., Knoblauch, D., \& Murphy, P. K. (2007). Academic motivation and achievement among urban adolescents. Urban Education, 42, 196-222.

Martin, A. J. (2006). The relationship between teachers' perceptions of student motivation and engagement and teachers' enjoyment of and confidence in teaching. Asia-Pacific Journal of Teacher Education, 34(1), 73-93.

Metz, K. E. (2008). Narrowing the gulf between the practices of science and the elementary school science classroom. The Elementary School Journal, 109(2), 138-161.

National Academy of Sciences, National Academy of Engineering, and Institute of Medicine, Rising above the gathering storm: energizing and employing America for a brighter economic future (Washington, DC: The National Academies Press, 2007), pp. 1-591.

Nord, C., Roey, S., Perkins, R., Lyons, M., Lemanski, N., Brown, J., \&Schuknecht, J. (2011). The Nation's Report Card [TM]: America's High School Graduates. Results of the 2009 NAEP High School Transcript Study. NCES 2011-462. National Center for Education Statistics.

PyMOL: The PyMOL Molecular Graphics System, Version 2.0 Schrödinger, LLC.

Vedder-Weiss, D., \& Fortus, D. (2012). Adolescents' declining motivation to learn science: A follow-up study. Journal of Research in Science Teaching, 49(9), 1057-1095. doi:10.1002/tea.21049

Wu, H. K., \& Shah, P. (2004). Exploring visuospatial thinking in chemistry learning. Science

education, 88(3), 465-492. 